\title{
COINCIDENCE THEORY FOR INFINITE DIMENSIONAL MANIFOLDS
}

\author{
BY K. K. MUKHERJEA
}

Communicated by S. Smale, January 9, 1968.

1. Introduction. The classical Lefschetz fixed point theorem states that with each compact self-mapping $f$ of a metrizable ANR one can associate a number $L(f)$, the Lefschetz number of $f$, such that if $L(f)$ is nonzero, $f$ has a fixed point.

If the ANR in question happens to be a smooth Banach manifold and $f$ a smooth map with isolated fixed points, we can attach to each fixed point an index equal to \pm 1 or zero, and the sum of these indices is equal to $L(f)$. In other words, $L(f)$ is the algebraic number of fixed points of $f$. See $[1, \S 11]$.

Here we announce a coincidence point theorem for certain classes of Banach manifolds and certain classes of maps, which extends the Lefschetz-Fuller coincidence theorem [3] and which specializes to give the Lefschetz fixed point theorem for Banach manifolds. The theorem is formulated in terms of an $(\infty-p)$-dimensional cohomology theory for certain Banach manifolds.

These results form part of the author's Cornell doctoral dissertation, written under the direction of J. Eells. Full details will appear elsewhere. We use notation and terminology of [1].

2. $(\infty-p)$-dimensional cohomology. In what follows, $E$ will denote a $C^{\infty}$-smooth, separable Banach space. All Banach manifolds in consideration will be $C^{\infty}$-smooth, paracompact and such that their tangent bundles admit a reduction to $G L_{c}(E)$.

A basic tool is the following generalization of a lemma of PalaisŠvarc $[1, \S 1]$ :

Theorem 1. Let $X$ be a Banach manifold. Then there exists a nested sequence of closed submanifolds $\left\{X_{n}\right\}_{n \geq 1}: X_{n} \subset X_{n+1}, \operatorname{dim} X_{n}=n$, such that if $X_{\infty}=U_{n \geq 1} X_{n}$ with the direct limit topology, the natural inclusion map $i_{\infty}: X_{\infty} \rightarrow X$ is a homotopy equivalence.

Given a Banach manifold $X$, choose a system $X_{1} \subset X_{2} \subset \cdots \subset X_{n}$ $\subset \cdots \subset X$ as described in Theorem 1. For any abelian group we define the $(\infty-p)$-dimensional cohomology of $X$ with compact supports with coefficients in $G$, denoted by $H_{0}^{\infty-p}(X, G)$, as follows: for all $n \geqq p \geqq 0$, we have

$$
\alpha: H_{c}^{n-p}\left(X_{n} ; G\right) \rightarrow H_{c}^{n+1-p}\left(X_{n+1} ; G\right)
$$


given by the composition

$$
H_{c}^{n-p}\left(X_{n}, G\right) \stackrel{\cong}{\rightarrow} H_{p}\left(X_{n} ; G\right) \rightarrow H_{p}\left(X_{n+1} ; G\right) \stackrel{\cong}{\rightarrow} H_{c}^{n+1-p}\left(X_{n+1}, G\right)
$$

where $H_{*}$ is singular homology, $H_{c}^{*}$ is singular cohomology with compact supports and twisted coefficients and the isomorphisms arise from Poincaré duality. $\left\{H_{c}^{\infty-p}\left(X_{n} ; G\right), \alpha\right\}$ is a direct system; we define its limit to be $H_{c}^{\infty-p}(X, G)$.

From Theorem 1, it follows that Poincare duality holds, i.e.

$$
H_{p}(X, G) \stackrel{\approx}{\rightarrow} H_{0}^{\infty-p}(X, G)
$$

and hence $H_{c}^{\infty-*}(X, G)$ is independent of the family $\left\{X_{n} \subset X\right\}$ used to define it.

It can be shown that if $f: X \rightarrow Y$ is a $C^{\infty}$-Fredholm proper map of index zero, provided $f$ respects orientation, there is an induced map

$$
f^{*}: H_{o}^{\infty-*}(Y, G) \rightarrow H_{o}^{\infty-*}(X, G) .
$$

ReMARKs. 1. The term "compact supports" used in describing $H_{o}^{\infty-*}$ is an abuse of terminology. The supports are not compact sets as is easily seen by considering the case of open sets in Hilbert space. In this case, the supports are closed, bounded sets.

2. Since $H_{c}^{\infty-0}(X, Z)$ is always $\boldsymbol{Z}$, we can define as in the finite dimensional case, the degree of a proper Fredholm map of index zero. This degree is a "globalisation" of Leray-Schauder degree.

3. A somewhat similar finite codimensional cohomology theory was introduced by Gęba-Granas [4] for closed bounded subsets of a Banach space.

\section{Coincidence theorems. Examples.}

Theorem 2. Let $X, Y$ be Banach manifolds and $f, g: X \rightarrow Y$ maps satisfying

(i) $f$ is proper, $C^{\infty}$, Fredholm of index zero and $f$ oriented;

(ii) $\overline{\mathrm{g} X}$ is compact.

Let $\theta_{p}(f, g): H_{p}(X, Q) \rightarrow H_{p}(X, Q)$ be defined by the composition

$$
H_{p}(X, Q) \stackrel{g_{*}}{\rightarrow} H_{p}(Y, Q) \stackrel{\cong}{\rightarrow} H_{0}^{\infty-p}(Y, Q) \stackrel{f_{*}}{\rightarrow} H_{0}^{\infty-p}(X, Q) \stackrel{\cong}{\rightarrow} H_{p}(X, Q)
$$

where the isomorphisms arise from Poincare duality. Then $L(f, g)$ $=\sum_{p \geq 0}(-p)^{p} \cdot \operatorname{Tr} \theta_{p}(f, g)$ is defined. If $L(f, g) \neq 0$, there exists an $x \in X$ such that $f(x)=g(x)$. 
Moreover, if the coincidence points are isolated and regular points of $f$, one can attach an index to each coincidence point. $L(f, g)$ is then the sum of the indices of each coincidence point. The index depends only on the derivatives of $f$ and $g$ at the coincidence points.

The proof of Theorem 2 follows almost immediately from the definition of $f^{*}$ and the following finite dimensional coincidence theorem:

Theorem 3. Let $f, g: X \rightarrow Y$ be maps between n-manifolds. Suppose the following conditions hold:

1. Given a compact set $K \subset X$ there is an n-manifold $M$ with boundary, such that $K \subset$ interior of $M$.

2. $f$ is proper; $\overline{g X}$ is compact and $f$ oriented.

Let $\theta_{p}: H_{p}(X: Q) \rightarrow H_{p}(X: Q)$ be the composition:

$$
H_{p}(X, Q) \stackrel{g_{*}}{\rightarrow} H_{p}(Y, Q) \stackrel{\cong}{\rightarrow} H_{0}^{n-p}(X, Q) \stackrel{f_{*}}{\rightarrow} H_{0}^{n-p}(X ; Q) \stackrel{\cong}{\rightarrow} H_{p}(X, Q)
$$

where the isomorphisms arise from Poincare duality. Then $L(f, g)$ $=\sum_{p \geq 0}(-1)^{p} \operatorname{Tr}\left(\theta_{p}\right)$ is defined. There is a proper homotopy of $f$ which frees coincidences with $g$ iff $L(f, g) \neq 0$.

For differentiable manifolds and maps, we may assume without loss of generality that the coincidence points are isolated and are regular points of $f$. If $\alpha \in X$ is such a coincidence point, let $i(\alpha)$-the index of $\alpha$-be

$$
\operatorname{Sign}\left(\operatorname{det}\left(f_{*}(\alpha)-g_{*}(\alpha)\right)\right) \text {. }
$$

Then $L(f, g)=\sum i(\alpha)$, summed over all the coincidence points.

N.B. Condition 1 always holds for differentiable manifolds.

EXAmple 1. Let $U \subset E$ be a convex set, $X$ an $E$-manifold; $f: U \rightarrow X$ a proper Fredholm map of zero index and nonzero degree. Then $f$ has coincidences with any compact map $g: X \rightarrow E$.

EXAMPLE 2. Let $P(H)$ be the projective space of $H$, a separable Hilbert space; $X$ an $H$-manifold. If $f: X \rightarrow P(H)$ is a Fredholm proper map of zero index and nonzero degree, then $f$ has coincidences with any compact map $g: X \rightarrow P(H)$.

4. On the proofs of Theorems 1 and 3.

Proof of Theorem 1. A result of Elworthy [2] implies that for Banach manifolds satisfying the conditions laid down, there exists a proper Fredholm map $\phi: X \rightarrow E$ which is surjective.

Now choose a sequence of finite dimensional subspaces:

$$
E_{1} \subset E_{2} \subset \cdots \subset E_{n} \subset \cdots \subset E
$$

whose union is dense in $E$. 
By altering the imbeddings $E_{n} \rightarrow E$ slightly, we can ensure that they are transversal to $\phi$, using a theorem of Smale [5]. Lifting back to $X$, we get closed oriented submanifolds

$$
X_{1} \subset X_{2} \subset \cdots \subset X_{n} \subset \cdots \subset X .
$$

It is easily checked that if the imbeddings are carefully chosen, the $X_{n}$ 's will be such that any compact $K \subset X$ is contained in a tubular neighbourhood of some $X_{n}$. Therefore $X_{\infty} \rightarrow X$ is a weak homotopy equivalence. Recalling that $X$ is an ANR yields the result, by a standard argument involving Whitehead's theorem.

Proof of Theorem 3. Condition 1 implies that a coincidence freeing proper homotopy of $f$ exists if and only if a coincidence freeing homotopy exists, which is constant everywhere outside some compact set contained in $X$. The proof now follows from obstruction theory using arguments similar to those employed in [3].

\section{REFERENCES}

1. J. Eells, $A$ setting for global analysis, Bull. Amer. Math. Soc. 72 (1966), 751-807.

2. K. D. Elworthy, Fredholm maps and $\mathrm{GL}_{\mathrm{c}}(E)$-structures, Bull. Amer. Math. Soc. 74 (1968), 582-586.

3. E. Fadell, On a coincidence theorem of F. B. Fuller, Pacific J. Math. 15 (1965), 825-834.

4. K. Gęba and A. Granas, Algebraic topology in linear normed spaces. III, Bull. Acad. Polon. Sci. Sér. Sci. Math. Astr. Phys. 15 (1967), 137-143.

5. S. Smale, $A n$ infinite dimensional version of Sard's theorem, Amer. J. Math. 87 (1965), 861-866.

Cornell University 